\title{
Construcción y Validación de un Sistema Web/móvil como Herramienta de Intervención Tecnológica para la Evaluación Dinámica en Asignatura de Grado en Ingeniería en Sistemas de Información
}

\section{Ramiro Walter Garbarini}

Grupo de Estudio en Metodologías de Ingeniería de Software. Facultad Regional Buenos Aires. Universidad Tecnológica Nacional, Argentina

rgarbarini@frba.utn.edu.ar

\section{Pablo Cigliuti}

Grupo de Estudio en Metodologías de Ingeniería de Software. Facultad Regional Buenos Aires. Universidad Tecnológica Nacional, Argentina pabcig@gmail.com

\section{Paola Saavedra Martínez}

Grupo de Estudio en Metodologías de Ingeniería de Software. Facultad Regional Buenos Aires. Universidad Tecnológica Nacional, Argentina psaavedra_martinez@hotmail.com

\section{Laura Recchini}

Grupo de Estudio en Metodologías de Ingeniería de Software. Facultad Regional Buenos Aires. Universidad Tecnológica Nacional, Argentina Irecchini@gmail.com

\section{Ma. Florencia Pollo}

Grupo de Estudio en Metodologías de Ingeniería de Software. Facultad Regional Buenos Aires. Universidad Tecnológica Nacional, Argentina

flo.pollo@gmail.com

\section{Yasnalla Rivero Peña}

Departamento de Desarrollo de Sistemas de Información y Tecnologías Web. Dirección de Tecnologías de la Información. Universidad de Holguín, Cuba yasnalla@uho.edu.cu 


\title{
Ezequiel Gaspes
}

Universidad Nacional de La Plata, Ezequiel@ecologix.com.ar

\section{Gustavo Osuna}

Universidad de Buenos Aires, gustavo_osuna@itrsa.com.ar

Recepción 18/11/2016

Aprobación 20/07/2017

\section{Resumen}

La aplicación de tecnología educativa en los dispositivos didácticos se ha incrementado en los últimos años. El objetivo del proyecto fue construir y validar un artefacto software, web y móvil, para la evaluación dinámica en asignatura de grado y de esta forma intervenir positivamente en la didáctica. En el presente artículo se describe la experiencia llevada a cabo, durante el primer semestre del año 2016, en cuatro (4) cursos de la asignatura Análisis de Sistemas, correspondiente al segundo nivel de la carrera de Ingeniería en Sistemas de Información de la Facultad Regional Buenos Aires. Teniendo en cuenta que la población objeto posee afinidad y habilidad para la adopción y utilización de nuevas tecnologías, el producto resultante busca facilitarle a la misma el proceso de repaso y autoevaluación, así como brindarles a los docentes información sobre el estado de dicho proceso. La puesta en práctica del mismo, forma parte del siguiente paso en la línea de acción del Grupo GEMIS de cara al futuro.

Palabras clave: M-learning; Análisis de Proceso de Evaluación; Tecnología informática aplicada a la educación.

\begin{abstract}
The application of educational technology in didactic devices has increased in recent years. The objective of the project was to construct and validate a software, web and mobile artifact for the dynamic evaluation in subject of degree and in this way to intervene positively in didactics. This article describes the experience carried out during the first semester of 2016 in four (4) courses in Systems Analysis, corresponding to the second level of the Information Systems Engineering degree at the Regional Faculty Buenos Aires. Taking into account that the target population has an affinity and ability to adopt and use new technologies, the resulting product seeks to facilitate the process of review and self-evaluation, as well as providing teachers with information on the status of said process. The implementation of it, is part of the next step in the line of action of the GEMIS Group for the future.
\end{abstract}

Keywords: M-learning; Analysis of Evaluation Process; Computer technology applied to education 


\section{Introducción}

Las nuevas tecnologías de la información y las comunicaciones, abren la posibilidad de disponer de dispositivos didácticos intervenidos tecnológicamente que ofrezcan al estudiante alternativas para el estudio y seguimiento de los contenidos y la posibilidad de realizar actividades académicas desde diversos espacios físicos y temporales; permitiéndole además al docente, documentar la trazabilidad de las interacciones de los alumnos y de los resultados obtenidos.

En este contexto, y a partir de la propuesta del Grupo GEMIS para desarrollar una aplicación que facilite la evaluación dinámica en asignatura de grado en Ingeniería en Sistemas de Información (UTN-FRBA, 2008), se decidió construir un sistema software basado en dicha propuesta y validarlo mediante su utilización durante el primer semestre del año 2016, en 4 (cuatro) cursos de la asignatura Análisis de Sistemas (UTN-FRBA, 2007). El sistema desarrollado, nombrado Sistema Cuestionarios On-Line, pretende ser una herramienta de repaso, entrenamiento y puesta a prueba de la profundidad que efectivamente cada alumno tiene sobre el manejo de los temas y conceptos desarrollados previamente en clase.

Al mismo tiempo, se espera que el acceso de los alumnos a los resultados obtenidos logre motivarlos y los impulse a un estudio continuo de los contenidos de la asignatura correspondiente. De igual manera, para los docentes pretende ser una herramienta de seguimiento permanente obteniendo alertas tempranas por alumno y por temas que presenten mayor dificultad para los estudiantes.

\section{Desarrollo}

El objetivo general del trabajo fue desarrollar un Sistema Cuestionarios On-Line (web / móvil) para favorecer el proceso de enseñanza-aprendizaje. Los primeros pasos del proyecto se centraron en definir la visión del proyecto pretendiendo que el sistema brinde información y herramientas a:

i. los docentes custodiar el complejo proceso de enseñanza aprendizaje y detectar a tiempo los problemas que puedan llegar a tener los alumnos durante la cursada de la asignatura y no al finalizar la misma. Este diagnóstico prematuro permitirá a los docentes realizar una mejor gestión del aula, analizando la información que brinde el uso del producto software (A. Deroche et al., 2015).

ii. a los alumnos, que podrán consultar los resultados alcanzados, valorar su aprendizaje y medir el grado de conocimiento adquirido por cada tema de la asignatura de forma supervisada.

\section{Etapas de trabajo}

Etapa A - Planificación. A partir de la visión definida se estableció el alcance de la solución, la cual está definida por la construcción de 2 (dos) productos: 
a. Sistema Cuestionarios Web, el cual cuenta con tres módulos destinados a (i) Administrador, su finalidad es la gestión de seguridad, la de usuarios y de los parámetros generales del sistema (ii) Docentes, destinado principalmente para la generación y publicación de cuestionarios, seguimiento y reportes; (iii) Alumnos, para responder cuestionarios y obtener reportes a partir de los resultados obtenidos.

b. Sistema Cuestionarios Móvil, solo destinado para que los alumnos respondan los cuestionarios publicados.

Luego se elaboró el plan director para la construcción del sistema, donde se definieron las siguientes etapas y sus resultados esperados.

\begin{tabular}{|l|l|}
\hline Etapa & \multicolumn{1}{|c|}{ Descripción } \\
\hline A & Planificación \\
\hline A.1 & Definición y análisis del proyecto \\
\hline A.2 & $\begin{array}{l}\text { Definición del alcance y metodología del desar- } \\
\text { rollo }\end{array}$ \\
\hline A.3 & Desarrollo del Plan Director \\
\hline B & Análisis \\
\hline B.1 & $\begin{array}{l}\text { Especificar Requerimientos Funcionales y No } \\
\text { Funcionales }\end{array}$ \\
\hline B.2 & Definición del modelo conceptual de datos \\
\hline C & $\begin{array}{l}\text { Construcción } \\
\text { Line (Diseño, Desarrollo, pruebas unitarias, } \\
\text { pruebas funcionales) }\end{array}$ \\
\hline C.1 & Construcción Sistema Cuestionarios Web \\
\hline C.2 & Construcción Sistema Cuestionarios Móvil \\
\hline D & $\begin{array}{l}\text { Validación del Sistema Cuestionarios On- } \\
\text { Line. }\end{array}$ \\
\hline D.1 & $\begin{array}{l}\text { Implementación del Sistema en entorno produc- } \\
\text { tivo }\end{array}$ \\
\hline D.2 & $\begin{array}{l}\text { Ejecución de la validación en cursos de Análisis } \\
\text { de Sistemas }\end{array}$ \\
\hline D.3 & $\begin{array}{l}\text { Ejecución y análisis de encuesta de opinión } \\
\text { respecto a la utilización del sistema }\end{array}$ \\
\hline
\end{tabular}

Tabla 1. Etapas del Proyecto.

\begin{tabular}{|l|l|}
\hline Etapa & Resultados Esperados \\
\hline A & Plan Director \\
\hline B & $\begin{array}{l}\text { Requerimientos Especificados } \\
\text { Modelo conceptual de datos }\end{array}$ \\
\hline C & $\begin{array}{l}\text { Sistema Software diseñado, construido y } \\
\text { probados: }\end{array}$ \\
\hline D & Validación ejecutada. \\
\hline
\end{tabular}


El estado del plan de trabajo es el siguiente:

\begin{tabular}{|l|l|}
\hline Etapa & Estado de Avance \\
\hline A & Finalizada \\
\hline B & Finalizada \\
\hline C & Finalizada \\
\hline D & Finalizada \\
\hline
\end{tabular}

Etapa B - Análisis. A continuación, se presentan extractos de los resultados obtenidos en esta etapa:

\section{Requerimientos Funcionales}

\section{Módulo Administrador}

1) Gestión de Parámetros Generales

a) Mantenimiento de parámetros: tiempo de respuesta, nivel de dificultad, tipo de cuestionario, etc.

2) Gestión de Seguridad y Usuarios

a) Altas, bajas y modificaciones de Docentes y Alumnos.

b) Seguridad

3) Gestión de Asignaturas

a) Altas, bajas y modificaciones de Asignatura.

b) Asignación de Docentes a las Asignaturas

c) Altas, bajas y modificaciones de Temas de una Asignatura.

4) Gestión de Cursos por Asignatura

a) Altas, bajas y modificaciones de Cursos.

b) Asignación de Docentes y Alumnos a Cursos.

\section{Módulo Docente}

5) Gestión de Preguntas 
a) Altas, bajas y modificaciones de Preguntas.

6) Gestión de Cuestionarios

a) Altas, bajas y modificaciones de Cuestionarios.

b) Asignación de Preguntas a Cuestionarios.

c) Publicación de Cuestionarios.

7) Reportes

a) Generación de reportes por tema, cuestionario, curso.

\section{Módulo Alumno Web}

8) Cursos

a) Consulta de Cursos asignados.

b) Consulta de Ranking de Alumnos por Curso

c) Visualización de Cuestionarios publicados al Curso.

9) Cuestionarios

a) Listado de Cuestionarios asignados, discriminado por Tipo de Cuestionario, Estado y Fecha de Vigencia.

b) Responder Cuestionario, un Cuestionario puede responderse de forma total o parcial, pudiendo continuar en otro momento desde la primera pregunta sin visualizar.

b) Reporte de Cuestionario finalizado, se debe mostrar cada pregunta, su resultado y corrección, y un resultado resumen de todo el cuestionario.

\section{Módulo Sistema Preguntas On-Line Móvil - Alumno}

10) Cursos

a) Consulta de Cursos asignados.

b) Visualización de Cuestionarios publicados al Curso.

11) Cuestionarios

a) Listado de Cuestionarios asignados, discriminado por Tipo de Cuestionario, 
Estado y Fecha de Vigencia.

b) Responder Cuestionario, un Cuestionario puede responderse de forma total o parcial, pudiendo continuar en otro momento desde la primera pregunta sin visualizar.

c) Reporte de Cuestionario finalizado, se debe mostrar cada pregunta, su resultado y corrección, y un resultado resumen de todo el cuestionario.

\section{Modelo Conceptual de Datos}

El siguiente diagrama contiene las entidades más representativas del modelo de datos.

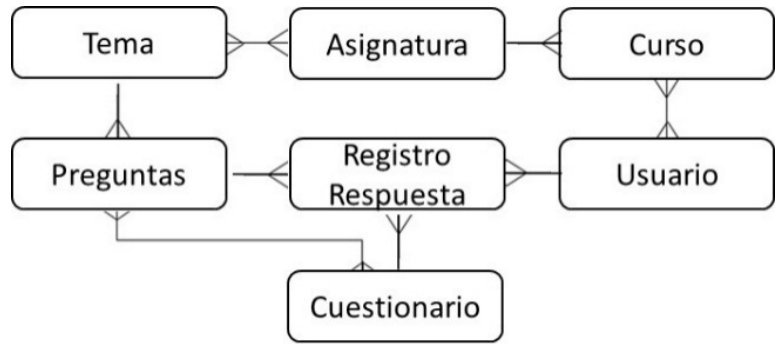

Figura 1. Modelo conceptual de datos.

Etapa C - Construcción Sistema Cuestionarios On-Line (Diseño, Desarrollo, pruebas unitarias, pruebas funcionales). El sistema web fue diseñado utilizando el patrón MVC (Model View Controller) ( Eckstein, 2007) dividiendo la aplicación en tres capas que permiten separar la capa de presentación (View) de la capa de negocios (Model) y el controlador (Controller) del paso de datos entre una capa y la otra.

El mismo fue desarrollado en el lenguaje de programación Java (JAVA, 2015) y MySQL (MYSQL, 2015) como base de datos. En las distintas capas se implementan diferentes frameworks para incrementar la productividad, performance, calidad, transparencia, reutilización de código e integración. Esto mismo permite proveer servicios para la seguridad, log-in y pruebas de la aplicación, construcción de ejecutables y reportes.

La arquitectura del sistema web está basada en la utilización de Spring (Spring, 2015) como framework de aplicación, log 4 j (Foundation, 2015a) para la generación de logs e informes de estados del sistema e identificación de alertas, Junit (JUnit, 2015) para pruebas unitarias y Maven (Foundation, 2015b) para la integración / build. 


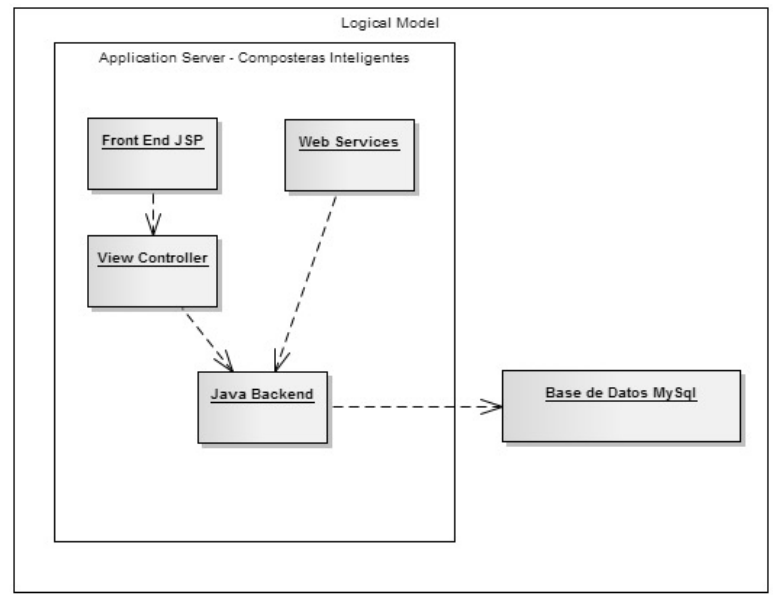

Figura 2.Modelo lógico.

La capa de presentación (front-end) WEB posee diversas características entre las cuales se destacan las siguientes:

- Las páginas son implementadas usando JSP y JavaScript.

- Spring es usado como Front Controller (Johnson, Hoeller, \& Donald, 2015) en la arquitectura MVC.

- JSP Tags usados para el contenido dinámico de páginas HTML.

- JSP Tag libraries son implementadas para reutilizar código.

- Las excepciones son manejadas para mostrar al usuario mensajes amigables.

- La inyección a servicios es usada con Spring framework.

- La capa de negocio es una implementación de servicios (web-services) para que el acceso a los datos tenga un tratamiento uniforme.

- Servicios para el manejo de excepciones garantizando el traslado de mensajes amigables ocultando los detalles de implementación.

El sistema móvil fue diseñado teniendo en cuenta un diseño previo de una aplicación móvil para asignatura de grado en ingeniería en sistemas de información (A.; Deroche et al., 2015), y fue desarrollado con tecnología phonegap (Adobe, 2016,). Dicho framework permite generar aplicaciones para celular con un frontend html y comunicación con el servidor de la misma manera que se hace desde un browser.

Como resultado de la sub etapa C.1, se obtuvieron el módulo Docente y el módulo Alumno. En particular, para el primero se presentan interfaces referidas a la gestión de preguntas y reportes. 


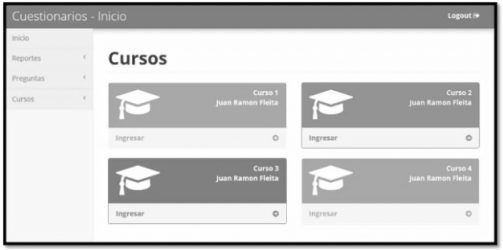

Figura 3. Interfaz para iniciar en el sistema.

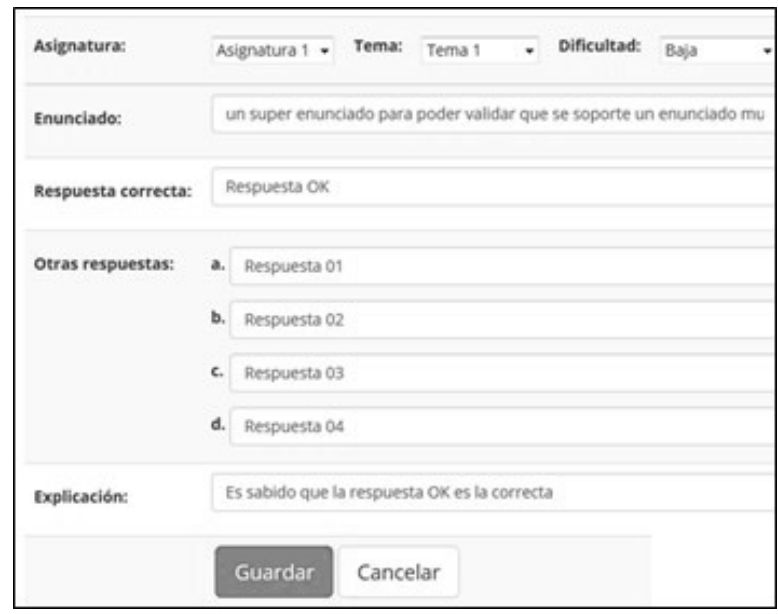

Figura 4.Interfaz para crear una pregunta.

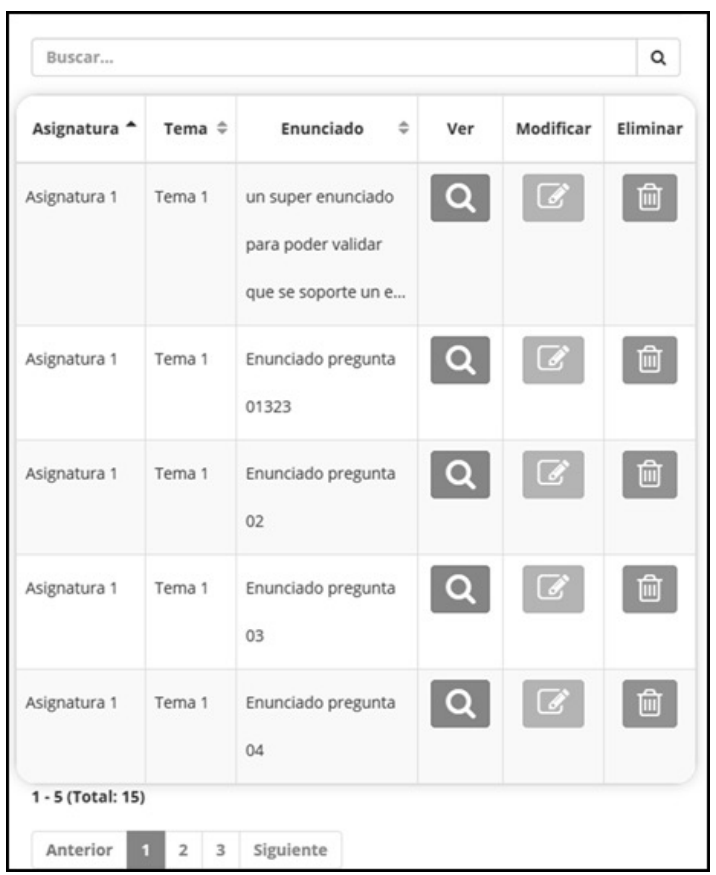

Figura 5. Lista de preguntas. 


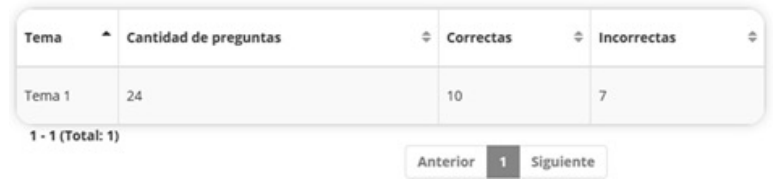

Figura 6. Reporte por tema.

\begin{tabular}{|l|l|l|l|}
\hline Curso & Cantidad de Preguntas & Correctas & $*$ Incorrectas \\
\hline Curso 1 & 12 & 10 & 7 \\
\hline Curso 2 & 0 & 0 & 0 \\
\hline Curso 3 & 0 & 0 & 0 \\
\hline Curso 4 & 0 & 0 & 0 \\
\hline 1.4 (Total: 4$)$ & \\
\hline Anterior & 1 siguiente
\end{tabular}

Figura 7. Reporte por curso.

El módulo Alumno es caracterizado por las pantallas para responder cuestionarios y visualizar los resultados obtenidos.

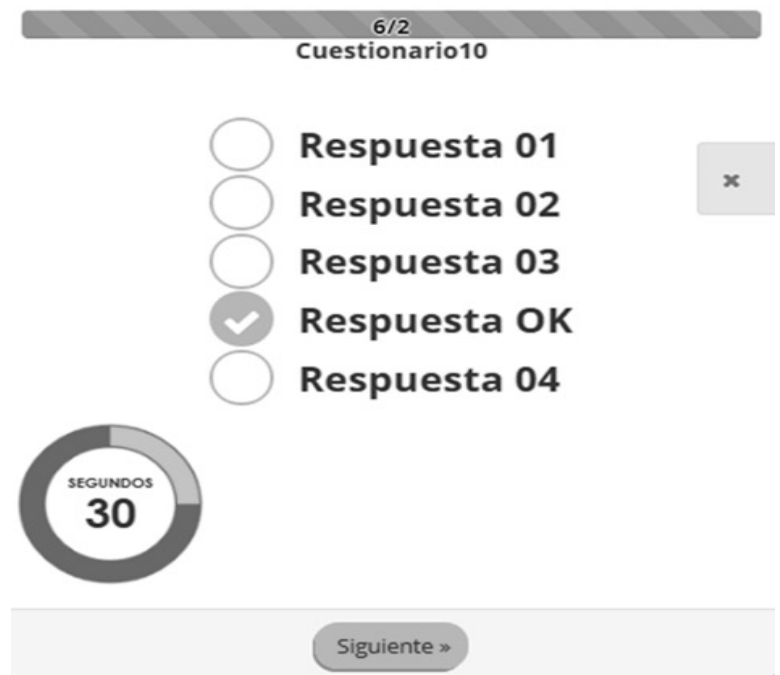

Figura 8.Interfaz para responder cuestionario. 


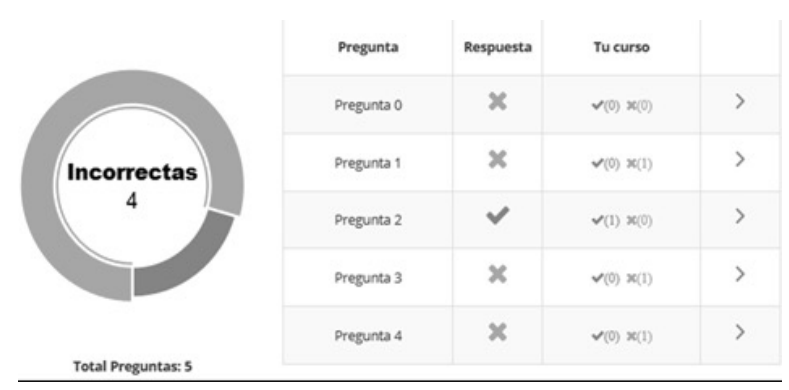

Figura 9. Resultados de los cuestionarios.

La sub etapa C.2, tuvo como resultado al Sistema Cuestionario Móvil destinado a los estudiantes.

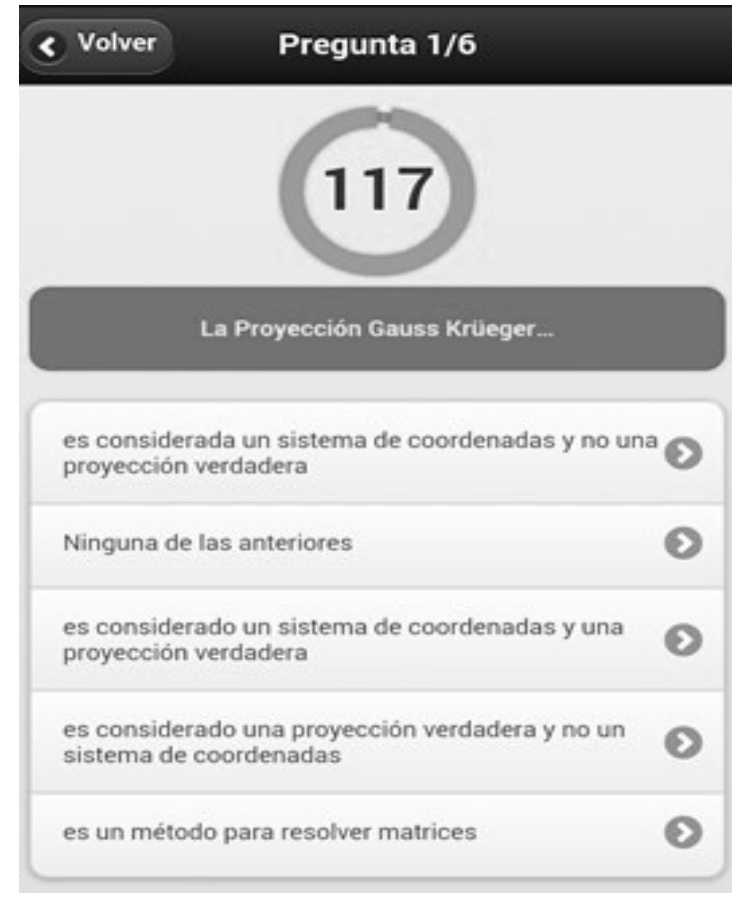

Figura 10. Responder cuestionario móvil. 


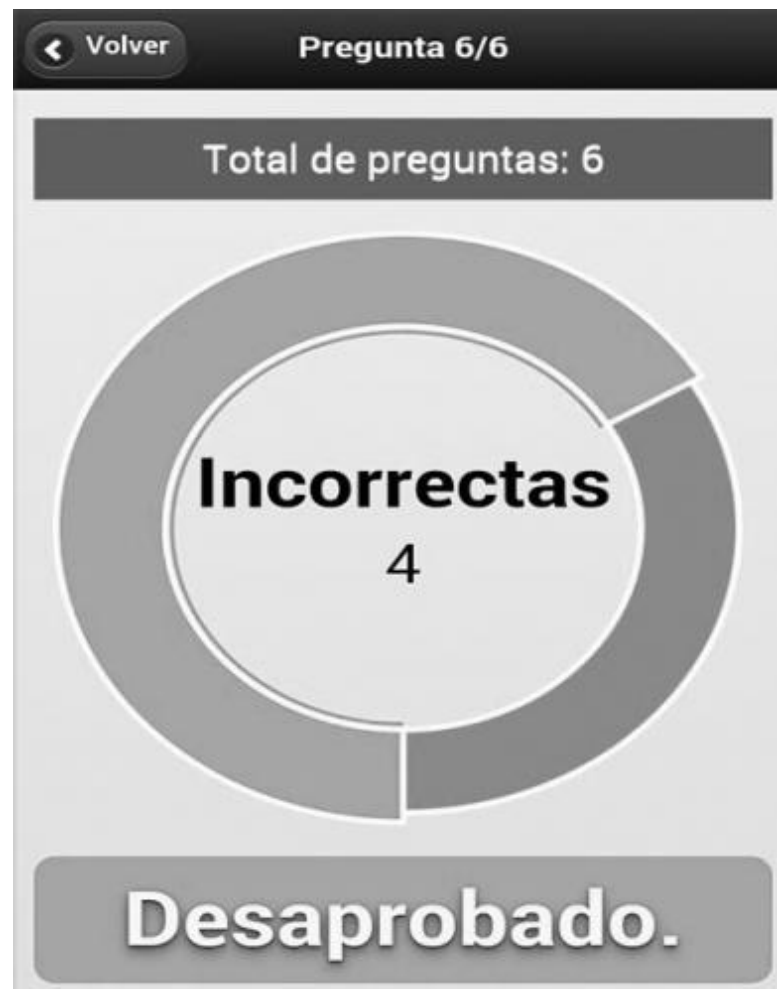

Figura 12.Resultados del cuestionario-móvil

Etapa D - Validación del Sistema Cuestionarios On-Line. El sistema fue implementado, etapa D.1, en el entorno cloud que la universidad posee basado en Microsoft Azure (Microsoft, 2016) y publicado para el acceso de los usuarios (GEMIS, 2016a), la aplicación móvil se encuentra disponible para ser descargada desde la web (GEMIS, 2016b).

Una vez corroborado el correcto funcionamiento del sistema en este entorno productivo, la sub-etapa D.2 se llevó adelante durante el primer cuatrimestre del ciclo lectivo 2016, en cuatro (4) cursos de la asignatura Análisis de Sistemas, para ello se crearon cien (100) preguntas, las cuales conformaron cuestionarios de dos (2) tipos (i) Seguimiento, publicados luego del desarrollo de un nuevo tema para que el docente pueda evaluar el grado de adopción del contenido desarrollado, y para que cada estudiantes pueda saber, de forma temprana, si ha comprendido el nuevo tema, (ii) Autoevaluación, son cuestionarios que integran diversos temas y son publicados previos a una evaluación, con la finalidad que los estudiantes tengan una instancia preliminar para medir sus conocimientos y poder reforzar los temas donde presentaron dificultades.

Como resultado de la ejecución de la sub-etapa D.2 se obtuvo que el 91,00\% de los estudiantes de los cursos objeto han participado respondiendo los cuestionarios publicados. En la siguiente tabla se detallan los cursos involucrados, la cantidad de estudiantes y el porcentaje de estudiantes que utilizaron el sistema respectivamente: 


\begin{tabular}{|c|c|c|c|}
\hline Curso & Turno & Cant.Estudiantes & Participación \\
\hline K-2.051 & Noche & 56 & $93,00 \%$ \\
\hline K-2.053 & Noche & 48 & $91,50 \%$ \\
\hline K-2.102 & Mañana & 42 & $90,50 \%$ \\
\hline K-2.104 & Mañana & 23 & $87,00 \%$ \\
\hline \multicolumn{2}{|c|}{ Total } & 169 & $91,00 \%$ \\
\hline
\end{tabular}

Tabla 4. Cursos involucrados.

La sub-etapa D.3, constó de la realización de una encuesta destinada al cuerpo docente y a los estudiantes para conocer sus opiniones respecto de la utilidad del sistema (Gemis, 2016a) . Los principales resultados obtenidos son los siguientes:

- El 100\% del cuerpo docente ha respondido la encuesta.

- El 35\% de los estudiantes respondieron la encuesta distribuidas de la siguiente forma:

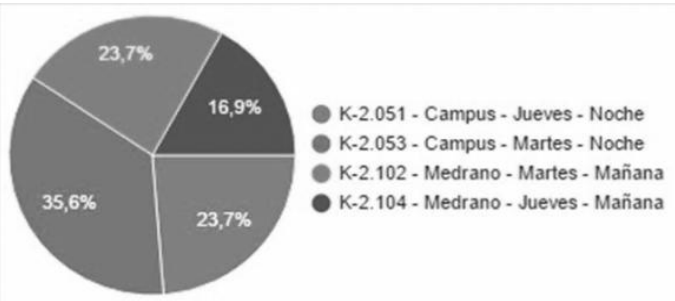

Tabla 5.Distribución de las respuestas según cursos

\begin{tabular}{|l|c|c|}
\hline Preguntas & Estudiantes & $\begin{array}{c}\text { Cuerpo } \\
\text { Docente }\end{array}$ \\
\hline $\begin{array}{l}\text { ¿Considera que el Sistema de Preguntas es una } \\
\text { herramienta útil para afianzar los conocimientos luego } \\
\text { de una clase? }\end{array}$ & $85 \%$ & $90 \%$ \\
\hline $\begin{array}{l}\text { El Sistema de Preguntas ayuda a medir el grado de } \\
\text { conocimiento sobre los temas de la asignatura. }\end{array}$ & $75 \%$ & $95 \%$ \\
\hline $\begin{array}{l}\text { El Sistema de Preguntas permite conocer la evolución } \\
\text { del desempeño de un estudiante a lo largo del curso. }\end{array}$ & $72 \%$ & $100 \%$ \\
\hline $\begin{array}{l}\text { El Sistema de Preguntas es una herramienta útil para } \\
\text { realizar una Auto-Evaluación previa a un examen. }\end{array}$ & $84 \%$ & $95 \%$ \\
\hline $\begin{array}{l}\text { ¿Recomendaría utilizar el Sistema de Preguntas en } \\
\text { otros cursos? }\end{array}$ & $90 \%$ & $100 \%$ \\
\hline
\end{tabular}

Tabla 6. Porcentaje de puntos obtenidos sobre puntos posibles. 


\section{Resultados y discusión}

Como resultado del presente trabajo se ha construido un Sistema de Cuestionarios On-Line, el cual integra tecnología web y móvil a los efectos de facilitar la evaluación dinámica y el seguimiento permanente de la evolución de los estudiantes en asignatura de grado en Ingeniería en Sistemas de Información.

El Sistema de Cuestionarios On-Line, a partir del uso por parte de los estudiantes, genera métricas que permiten identificar de forma temprana a aquellos estudiantes que posean dificultades con ciertos contenidos, y aquellos temas que presenten mayor dificultad al conjunto de los alumnos. En consecuencia, el sistema brinda información complementaria a la gestión y seguimiento que los docentes realizan en sus cursos, permitiéndoles tomar acciones tempranas.

La obtención del Sistema de Cuestionarios On-Line implicó la construcción y validación de los siguientes productos software: Sistema Cuestionarios - Web; Sistema Cuestionarios - Móvil y la realización de pruebas de integración de todos estos componentes, y la ejecución de pruebas de validación de uso en cuatro (4) cursos de Análisis de Sistemas. El análisis de opinión arrojó resultados positivos por parte de los estudiantes y docentes participantes respecto a la utilidad del sistema y el 90\% de los estudiantes que respondieron recomendarían el uso del sistema en otros cursos.

El próximo paso es continuar con la utilización del sistema durante el segundo cuatrimestre 2016, realizar los ajustes del software que surjan del uso cotidiano del mismo y de las mejoras propuestas por estudiantes y docentes. Así mismo, se hará un análisis los resultados de los exámenes parciales del 2016 y de los resultados históricos con el objeto de determinar si la implementación del sistema tuvo un impacto positivo en el rendimiento académico. En segunda instancia se propone para el 2017 extender el uso del sistema a todos los cursos de la cátedra de Análisis de Sistemas, UTN - FRBA, y realizar un nuevo proceso de validación del sistema.

En los últimos años, a nivel global y a partir del impulso dado por los avances y disponibilidad de las nuevas tecnologías de la información permite a la comunidad académica tener acceso a sistemas software como complemento para el estudio y seguimiento de los contenidos de cada clase desde diversos espacios físicos y temporales.

\section{Conclusiones}

Como conclusión podemos sostener que el Sistema Cuestionarios On-Line es propicio para que (i) los estudiantes puedan afianzar los conocimientos luego de una clase, y realizar una Auto-Evaluación previa a un examen, (ii) los docentes tengan más información acerca la evolución del desempeño individual y en conjunto de los estudiantes a lo largo del curso, y de esta forma intervenir positivamente en la didáctica. 


\section{Referencias}

Adobe. (2016). Adobe Phonegap Retrieved 01/08/2016. Disponible en <http:// phonegap.com>.

Deroche, A., Acosta, M., Vegega, C., Bernal, T., L., Straccia, L., Pytel, P. y PolloCattaneo, M. F. (2015). "Diseño de Aplicación Móvil para Asignatura de grado en Ingeniería en Sistemas de Información". Paper en 3er. Congreso Nacional de Ingeniería Informática y Sistemas de Información.

Deroche, A., Acosta, M., Vegega, C., Bernal Tomadoni, L., Straccia, L., Pytel, P. y Pollo-Cattaneo, M. F. (2015). "Propuesta de Desarrollo de Aplicación Móvil para la Evaluación Dinámica en Asignatura de Grado en Ingeniería en Sistemas de Información". Paper en Proceedings XVII Workshop de Investigadores en Ciencias de la Computación.

Eckstein, E. (2007). "Java SE Application Design With MVC". Disponible en <http:// www.oracle.com/technetwork/articles/javase/index-142890.html>.

Emis, G. (2016). "Aplicación Móvil Cuestionarios". Recuperado

el 01/08/2016., disponible en <https://drive.google.com/ open?id=0B9D0KeY9bAArZHVIQ2ZEZm9ERkk>.

Foundation, T. A. S. (2015a). “Apache Log4j 2”. Recuperado el 01/08/2016. Disponible en <http://logging.apache.org/log $4 \mathrm{j} />$.

Foundation, T. A. S. (2015b). "Welcome to Apache Maven". Recuperado el 01/08/2016. Disponible en <http://maven.apache.org/>.

GEMIS. (2016a). Encuesta de Opinión sobre el uso del Sistema Cuestionarios OnLine Retrieved 01/08/2016, from https://docs.google.com/forms/d/18tYpZ782Jt6H_ dir2UnuQDE9FzR1dWOC8LPecInDl-0/viewform? $\mathrm{c}=0 \& \mathrm{w}=1$

GEMIS. (2016b). Sistema Cuestionarios On-Line Retrieved 01/08/2016, from http:// cuestionarios.sistemas.frba.utn.edu.ar

JAVA. (2015). JAVA Retrieved 1/08/2016., from http://www.java.com

Johnson, R., Hoeller, J., \& Donald, K. (2015). "Spring Web MVC framework", Spring Framework Reference Documentation. Retrieved from http://docs.spring. io/spring/docs/current/spring-framework-reference/html/mvc.html

JUnit. (2015). “JUnit About” Retrieved 01/08/2016., from http://www.junit.org/

Microsoft. (2016). Microsoft Azure Retrieved 01/08/2016, http://azure.microsoft.com

MYSQL. (2015). MYSQL Retrieved 01/08/2016., from http://www.mysql.com

Spring. (2015). Spring Retrieved 1/08/2016., from http://www.springframework. org

Ordenanza $\mathrm{N}^{\circ} 1150$ del Consejo Superior Universitario de la Universidad Tecnológica Nacional (2007). Buenos Aires.

UTN-FRBA (2008). Programa de la asignatura Análisis de Sistemas - Plan 2008. Retrieved from http://sistemas.frba.utn.edu.ar/index.php/academico/plan-k08 